\title{
In Vitro Characterization of Six Month Dosage Forms for a GnRH Antagonist
}

\author{
Susan D'Souza, ${ }^{1}$ Santos Murty, ${ }^{2}$ Jabar A. Faraj, ${ }^{3}$ and Patrick P. DeLuca ${ }^{4}$ \\ ${ }^{1}$ Sunovion Pharmaceuticals Inc., Marlborough, MA 01752, USA \\ ${ }^{2}$ Murty Pharmaceuticals, 518 Codell Drive, Lexington, KY 40509, USA \\ ${ }^{3}$ Evonik, 750 Lakeshore Parkway, Birmingham, AL 35211, USA \\ ${ }^{4}$ University of Kentucky College of Pharmacy, Lexington, KY 40536, USA
}

Correspondence should be addressed to Susan D’Souza; dr_ssdsouza@yahoo.com

Received 4 April 2014; Accepted 28 May 2014; Published 15 July 2014

Academic Editor: Maria Cristina Bonferoni

Copyright (C) 2014 Susan D'Souza et al. This is an open access article distributed under the Creative Commons Attribution License, which permits unrestricted use, distribution, and reproduction in any medium, provided the original work is properly cited.

\begin{abstract}
The objective of this study was to develop long-acting injectable dosage forms of Orntide, a peptide GnRH antagonist, to provide tailored release for 6-month duration. Using a polylactide homopolymer and the solvent extraction/evaporation method, three microsphere formulations (Formulations $A, B$, and $C$ ) were prepared at various drug loadings (11.85-15.79\%). The microspheres were characterized for particle size by laser diffractometry, surface morphology by scanning electron microscopy (SEM), and bulk density by tapping, as well as long-term in vitro drug release, mass loss and hydration at $37^{\circ} \mathrm{C}$, and short-term in vitro drug release at elevated temperatures $\left(51-59^{\circ} \mathrm{C}\right)$. Experiments at $37^{\circ} \mathrm{C}$ revealed that drug release was triphasic and occurred due to slow degradation of the polylactide polymer. Short-term in vitro release results indicated that drug release was diffusional. Application of the Higuchi equation to short-term release confirmed the temperature dependency of the diffusional rate constant. Using the rate constant and the Arrhenius equation, an $E_{a}$ value of $45 \mathrm{kcal} / \mathrm{mol}$ (Formulation A) and approximately $25 \mathrm{kcal} / \mathrm{mol}$ (Formulations B and C) was obtained for diffusional release. Study results suggest that by selection of an appropriate biodegradable polymer, injectable dosing forms that release drug for 6 months or longer can be developed.
\end{abstract}

\section{Introduction}

Several reports document the challenges faced by pharmaceutical researchers in the area of drug delivery of large molecules [1-5]. Undoubtedly, administration of large molecules like peptides and proteins in vivo is fraught with obstacles. Unlike most small molecules, peptides and proteins show poor oral absorption and bioavailability due to their large molecular weight and rapid degradation in the gastrointestinal (GI) tract [6]. Choosing an alternate route of administration (e.g., parenteral, nasal) to deliver these types of large molecules does not provide significant advantages over oral administration due to the fact that they are rapidly degraded in body fluids, resulting in a short-half life in vivo [7]. Since proteins and peptides are generally considered to be highly potent molecules requiring administration of small therapeutic doses, a frequent dosing regimen is needed to maintain constant and continuous blood levels over a period of time. Such type of dosing is neither suitable nor ideal and often results in nonadherence to therapy, patient compliance issues, and increased costs [8].

Peptide therapeutics like GnRH (gonadotropin receptor hormone) analogs have been a mainstay in the treatment of prostate cancer. Leuprolide, Triptorelin, Nafarelin, and so forth are examples of peptides that have been clinically used with a great degree of success [9]. Due to their high affinity towards $\mathrm{GnRH}$ receptors, administration of these synthetic peptides, also known as GnRH agonists, causes an initial stimulation of pituitary luteinizing hormone (LH) and follicle-stimulating hormone (FSH) secretion, which is followed by a sharp drop in the secretion of these hormones. The decreased secretion/inhibition of LH and FSH (medical or chemical castration) is desirable in the treatment of sex hormone dependent diseases like prostate cancer [10]. Thus, treatment with GnRH agonists offers various benefits as these synthetic analogs exhibit higher potency, affinity, 
and an improved half-life over native physiological GnRH. Clinically, however, administration of $\mathrm{GnRH}$ agonists leads to a well-documented side effect (testosterone flare) that is a consequence of the initial stimulation in pituitary $\mathrm{LH}$ [11]. Studies have shown that upon long-term treatment with GnRH agonists, the sharp spike in serum testosterone persisted up to 7 days in rats and 4 weeks in humans and could be correlated with an increase in tumor growth and worsening of symptoms in patients [12, 13]. Hence, researchers began to explore a new class of peptide $\mathrm{GnRH}$ analogs (antagonists) that could suppress the $\mathrm{GnRH}$ receptors while preventing the hormonal response frequently observed with GnRH agonists [14, 15].

Orntide acetate is a potent novel GnRH antagonist that has shown great potential in treatment of prostate cancer and precocious puberty [16]. As an antagonist, Orntide is able to competitively block $\mathrm{GnRH}$ receptors and induce chemical castration without producing the initial testosterone flare that is a characteristic of GnRH agonists. Therefore, administration of Orntide eliminates the testosterone surge, reduces associated side effects, and ensures an improvement in the quality of life of patients on GnRH analog therapy [17]. However, similar to other GnRH analogs, Orntide has a short biological half-life, requiring frequent injections to initiate and maintain testosterone suppression [18].

One approach to initiating and sustaining in vivo levels of $\mathrm{GnRH}$ antagonists like Orntide can be through the use of polymeric excipients as carriers of the peptide. Indeed, this strategy has been successfully adopted with some of the earliest marketed dosage forms of $\mathrm{GnRH}$ agonists (e.g., Leuprolide) that released drug for 1,3 , or 4 months $[19,20]$. Of the several polymeric excipients suitable for parenteral use, the polyester class of polymers has been extensively studied and most widely used. The polylactic (PLA) and polyglycolic (PGA) acid classes of polymers, along with their copolymers, poly(lactide-co-glycolide) (PLGA), are undeniably the most common excipients used to formulate small and large molecule drugs as they offer the possibility of sustaining drug release and enhancement of bioavailability and protect the encapsulated molecule from in vivo degradation $[21,22]$. Selecting a PLA, PGA, or PLGA polymer to formulate a peptidic drug delivery system provides several advantages, especially since this class of polymers has long been approved by the US Food and Drug Administration (FDA) for use in humans. In addition to low immunogenicity and low toxicity, these polymers are biodegradable and biocompatible, have a well-known and extensive safety profile, and are cleared in vivo by the Krebs cycle. Unsurprisingly, they are often the polymers most utilized to develop drug delivery systems like implants, nanospheres, or microspheres, designed to provide sustained release of small molecules and biologics intramuscularly or subcutaneously [23-27].

Another reason for the increasing use of PLA and PLGA polymers as excipients is that several properties can be altered to tailor drug release rate and maintain in vivo therapeutic levels for varying durations. For instance, studies have shown that the time taken for complete biodegradation of the polymer increases with increasing molecular weight, thereby increasing its in vivo residence time (i.e., increased duration of action) $[28,29]$. Similarly, by altering the ratio of lactide or glycolide in a PLGA copolymer, a wide range of degradation rates can be obtained. As an example, a 90:10 lactide: glycolide copolymer degrades more slowly than a $65: 35$ or $50: 50$ PLGA copolymer. This is because an increase in the more hydrophobic lactide moiety ensures a slower degradation rate of the PLGA polymer leading to extended duration of drug release [30-32]. Thus, the PLA homopolymer degrades much slower than a PGA homopolymer or PLGA copolymer.

From literature, it is well known that PLGA copolymers have been the excipients of choice to encapsulate $\mathrm{GnRH}$ agonists and antagonists that are used to treat prostate cancer [33-36]. However, several reports noted that therapy for 14 months was less than ideal, leading to the development and eventual approval of a dosage form that released the $\mathrm{GnRH}$ agonist (Leuprolide) for 6 months [37, 38]. In line with recent recommendations for the treatment of prostate cancer and in a manner similar to the findings noted with $\mathrm{GnRH}$ agonist therapy, extending Orntide release for 6 months would reduce the frequency of dosing and improve patient compliance $[39,40]$. Hence, the goal of the current study was to develop and investigate the suitability of a PLA polymer towards providing tailored release of Orntide for a period of 6 months. Several characterization studies involving assessment of drug content by HPLC, particle size by laser diffractometry, surface morphology by scanning electron microscopy (SEM), mass loss by gravimetry, and in vitro drug release profiles at real-time and elevated conditions were performed on three PLA microsphere formulations containing Orntide.

\section{Materials and Methods}

2.1. Materials. Orntide acetate was supplied by California Peptide Research, Inc. (Napa, CA), and was 99\% pure. Polylactide (PLA) polymer R202H was purchased from Boehringer Ingelheim. All solvents and other chemicals were of analytical grade.

2.2. Preparation of Microspheres. Three batches of Orntide microspheres were prepared by a solvent extraction/evaporation method and recovered by filtration [33]. Briefly, a solution of peptide in methanol was combined with a solution of PLA in methylene chloride and stirred until clear. The solution was then slowly injected into an aqueous continuous phase under stirring with a Silverson L4R mixer (Silverson machines, MA, USA) at a predetermined speed. Subsequently, the solvents were removed by stirring after which the microspheres were recovered by filtration, suspended in a suitable vehicle, filled into vials, and freeze-dried. The residual solvent levels were below ICH limits of $600 \mathrm{ppm}$.

\subsection{Characterization of Microspheres}

2.3.1. Drug Content. The peptide was analyzed by reverse phase high-performance liquid chromatographyHPLC 
(Bondclone $10 \mathrm{C}-18$ column, $150 \mathrm{~mm} \times 3.90 \mathrm{~mm}$ ). The elution phase consisted of $34 \%(\mathrm{v} / \mathrm{v})$ acetonitrile and $0.1 \%(\mathrm{v} / \mathrm{v})$ trifluoroacetic acid in water. UV detection was at $\lambda \max$ $215 \mathrm{~nm}$ [41]. Drug content (\%) was expressed as the "weight of drug in microspheres/weight of microspheres $\times 100$."

2.3.2. Particle Size. Particle size distribution of the microspheres prior to vialing was determined using a laser diffraction technique (Malvern 2600c Particle Sizer, Malvern, UK). The particles were suspended in $0.05 \%$ Tween 80 and counted using a laser sensor. The average particle size was expressed as volume mean diameter in microns $(\mu \mathrm{m})$.

2.3.3. Bulk Density. The dry microspheres were quantitatively transferred to a graduated test tube. The test tube was subsequently tapped 50 times from a vertical distance of approximately 0.5 inches and the occupied volume was recorded [8]. The tapping process was repeated until the volume occupied by particles remained unchanged. The final volume was recorded as bulk volume, $V_{b}$, and the tapped bulk density (g/cc) was calculated as $M / V_{b}$, where " $M$ " was the weight of microspheres employed.

2.4. In Vitro Release at $37^{\circ} \mathrm{C}$. Long-term in vitro release $\left(37^{\circ} \mathrm{C}\right)$ was performed using a modified dialysis method [41, 42]. Briefly, Orntide PLA microspheres were accurately weighed and placed in a $7 \mathrm{~mL}$ dialysis tube (Tube-O-Dialyzer, MWCO 300,000 Da) filled with 0.1 M Acetate buffer, pH 4.0, containing $0.1 \%$ sodium azide (inner media), which in turn was placed in a $50 \mathrm{~mL}$ tube containing $40 \mathrm{~mL}$ of the same release medium (outer media). The contents of the outer media were continuously stirred with a magnetic stirrer to prevent formation of an unstirred water layer at the outer dialyzing surface. At predetermined intervals, $1.0 \mathrm{~mL}$ samples was withdrawn from the outer media followed by buffer replacement with fresh buffer and HPLC analysis of peptide from the sampled outer medium.

2.5. In Vitro Release at Elevated Temperatures. Short-term (accelerated) in vitro release was performed by incubating an accurately weighed sample of Orntide PLA microspheres in $10 \mathrm{~mL}$ of $0.1 \mathrm{M}$ acetate buffer ( $\mathrm{pH} 4.0$ ) containing $0.5 \%$ polyvinyl alcohol (PVA) at temperatures above $50^{\circ} \mathrm{C}$. Sampling from the supernatant was carried out at specified intervals, after centrifugation at $3000 \mathrm{rpm}$ for $10 \mathrm{~min}$.

2.6. Degree of Hydration and Mass Loss. Hydration and mass loss experiments were performed at $37^{\circ} \mathrm{C}$. At predetermined time points, microspheres were recovered from the release medium and the wet mass was recorded $\left(W_{w}\right)$. The wet mass was dried for 48 hours under vacuum at room temperature and reweighed $\left(W_{d}\right)$.
TABLE 1: Properties of Orntide PLA microspheres.

\begin{tabular}{lccc}
\hline & \multicolumn{3}{c}{ Formulation } \\
& $\mathrm{A}$ & $\mathrm{B}$ & $\mathrm{C}$ \\
\hline Drug load $(\%)$ & 11.85 & 14.89 & 15.79 \\
Incorporation efficiency $(\%)$ & 79 & 71 & 88 \\
Bulk density $(\mathrm{g} / \mathrm{cc})$ & 0.49 & 0.50 & 0.45 \\
Mean particle size $(\mu \mathrm{m})$ & 14.9 & 14.7 & 15.9 \\
\hline
\end{tabular}

The degree of hydration and mass loss was calculated as follows:

$$
\begin{gathered}
\text { Degree of Hydration }=\frac{\left(W_{w}-W_{d}\right)}{W_{d}} \times 100, \\
\% \text { Mass Loss }=\frac{\left(W_{i}-W_{d}\right)}{W_{d}} \times 100,
\end{gathered}
$$

where $W_{i}=$ initial mass.

\section{Results and Discussion}

3.1. Characterization of Microspheres. Table 1 details the physical properties of Formulations $A-C$. Drug content, as determined by HPLC, ranged from 11.85 to $15.79 \%$ for the three formulations while incorporation efficiency of the peptide into the PLA polymer was between 70 and $90 \%$. The three formulations had intermediate bulk density values, around $0.50 \mathrm{~g} / \mathrm{cc}$, and similar mean particle size, indicating that in vitro performance of the formulations would be broadly similar.

3.2. In Vitro Release at $37^{\circ} \mathrm{C}$. Results of long-term (real-time) in vitro release at $37^{\circ} \mathrm{C}$ are presented in Figure 1 . From the figure, a very low initial burst (less than $3 \%$ by day 1 ) was observed for all the formulations. After the initial burst, drug release continued slowly to reach approximately $10 \%$ in 15 days. This slow release behavior was maintained through day 60 , where drug release was a modest $20-28 \%$. From day 60 to day 90 , release rate had increased to deliver nearly $35-$ $45 \%$ of the drug from the microspheres to the outer medium. This trend continued through day 120 , where nearly $75-85 \%$ release was noted. Subsequently, the release rate of Orntide slowed slightly and complete release was achieved by day 180 (6 months). Overall, the three formulations released drug in a similar fashion.

The data from Figure 1 provides an interesting insight into the mechanism of Orntide release from PLA microspheres. Indeed, the results from Figure 1 illustrate a drug release profile that is categorized as triphasic (sigmoidal) and has been observed previously with PLGA microspheres [42]. The three components of a triphasic release profile include initial burst, diffusional release, and erosional release. Initial burst occurs when drug that is loosely bound to the polymer surface or internal pores is easily leached and undergoes dissolution to enter the release media. This phase of drug release does not occur with drug that is encapsulated inside the polymer. After the initial burst release of drug, the next 


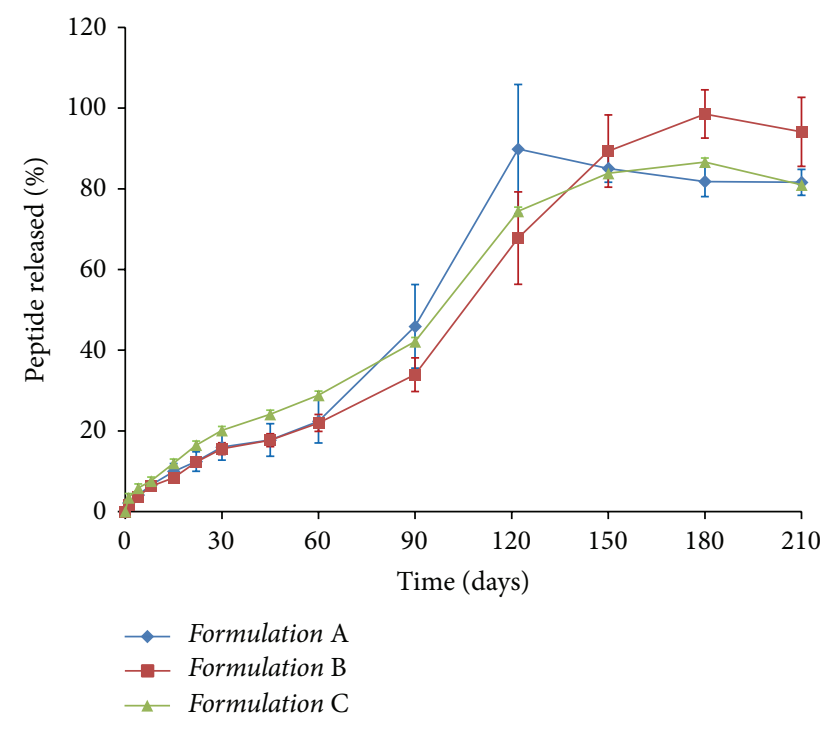

FIgURE 1: In vitro release of Orntide PLA microspheres at $37^{\circ} \mathrm{C}$.

phase involves dissolution of drug incorporated inside the polymer matrix and its transport (diffusion) to the release medium. This phase of drug release occurs when the polymer is being hydrated by the release media or body fluids and is typically characterized by low drug release rates. Continued polymer hydration leads to a phenomenon known as bulk hydrolysis of the ester bonds in PLA or PLGA and entails rapid polymer degradation, erosion, and loss of polymer mass leading to the final phase of drug release known as erosional release [43]. As such, due to the chemical nature of the polymer, drug release rates during the hydrolytic degradation phase (erosional phase) are typically much faster than those observed during the diffusional phase. From Figure 1, a low initial burst was observed at day 1 , with diffusional release through day 60, after which erosional release ensued and continued through day 180.

3.3. Degree of Hydration. Water incursion into the PLA polymer leads to a series of events, including drug release, hydration, and mass loss. Figure 2 shows the hydration data for the Orntide PLA microspheres. Orntide is a water-soluble peptide, while PLA is a water-insoluble polymer. The data in Figure 2 indicate that the polymer matrix was well hydrated by day 15 , corresponding to the diffusional release phase of the microspheres. The degree of hydration for Formulations $B$ and $C$ appeared to be similar, while Formulation $A$ lagged slightly. In general, hydration values from day 15 to day 60 ranged between 0.75 and 1.00 and were similar, indicating that the polymer was continuing to imbibe water and slowly release drug during the diffusional phase of release. By day 90 , hydration values had dropped to 0.50 , almost half of that observed at day 15. From Figure 1, this corresponds with the erosional phase of drug release. During this phase, the polymer chains undergo rapid and autocatalytic bulk hydrolysis leading to breakdown of ester bonds and formation of smaller chain fragments. The hydration data at day

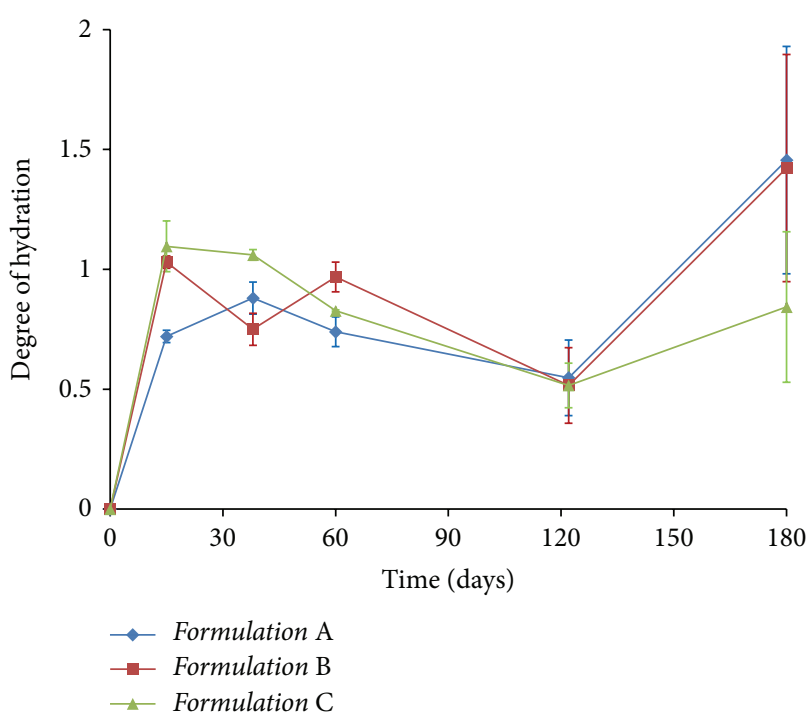

Figure 2: Hydration of Orntide PLA microspheres at $37^{\circ} \mathrm{C}$.

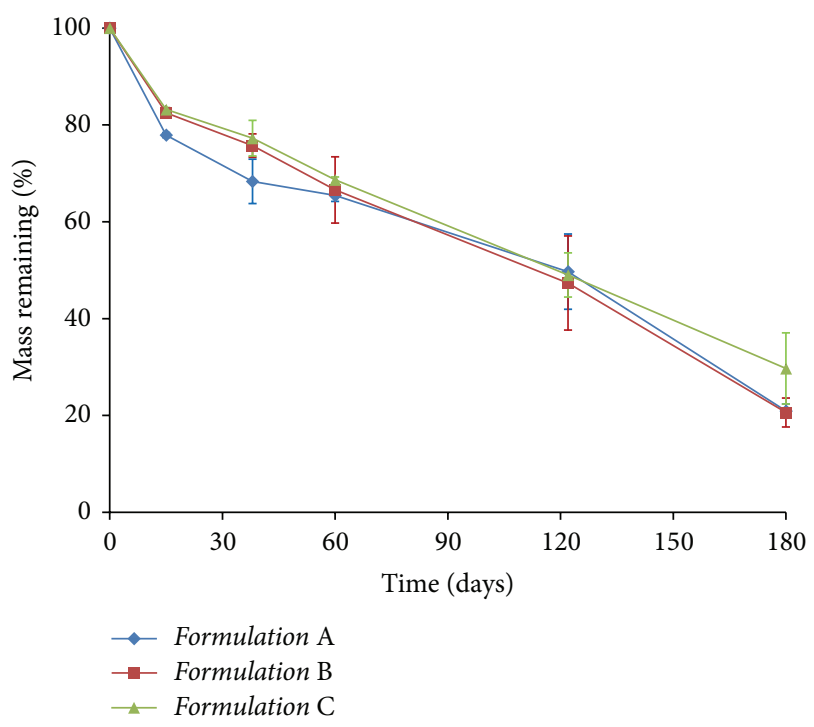

Figure 3: Mass loss of Orntide PLA microspheres at $37^{\circ} \mathrm{C}$.

120 suggest that the polymer is no longer imbibing water as rapidly as during the diffusional phase; rather, the polymer chains are sufficiently hydrated ensuring rapid ester bond hydrolysis. By day 180 , hydration values ranged between 0.84 and 1.45 , indicating that along with complete drug release due to polymer erosion, the remnant polymer fragments in the matrix were more hydrophilic than the original PLA.

3.4. Mass Loss. The amount of mass remaining in the Orntide PLA microspheres as a function of time is presented in Figure 3. In general, the mass loss profiles for the three formulations were similar. At the 15-day time point, mass loss was approximately $15-20 \%$ and rose steadily to about $35 \%$ by day 60 . Thus, the cumulative mass loss during the initial burst and diffusional phases of drug release was $34 \%$. Subsequently, 


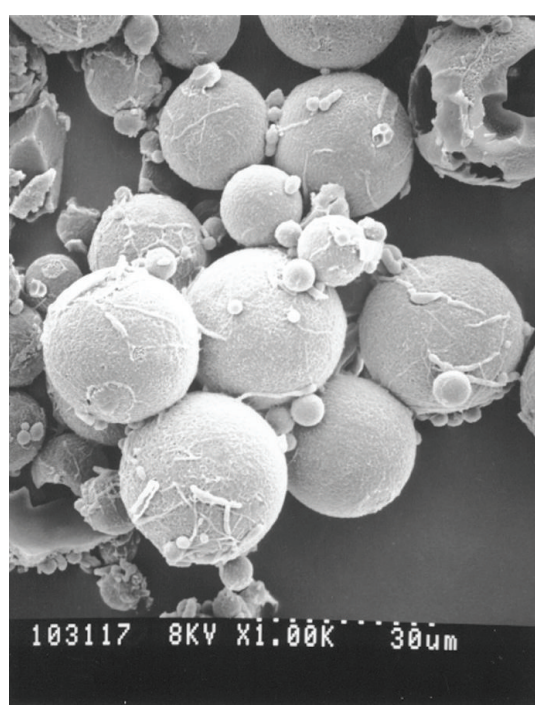

(a)

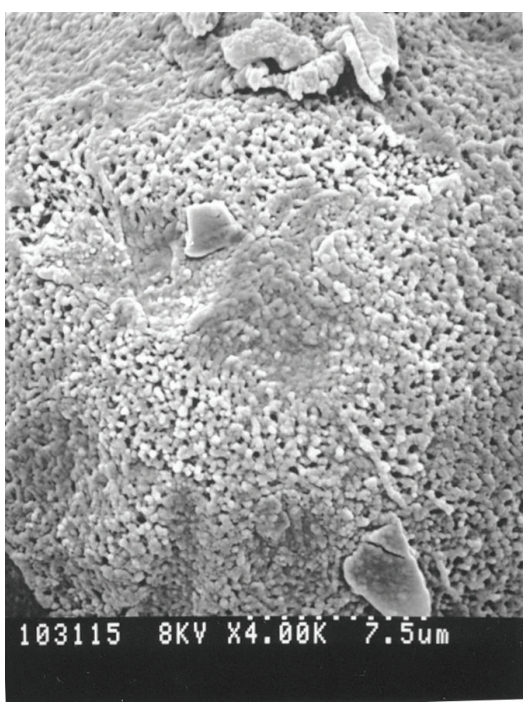

(b)

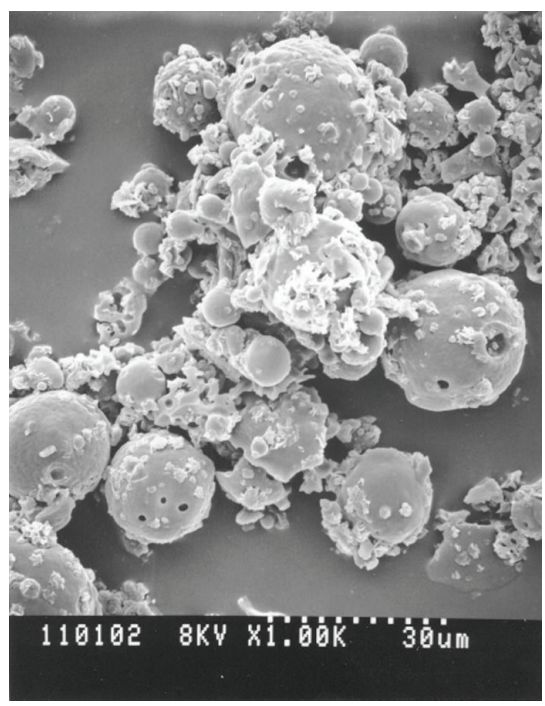

(c)

FIGURE 4: SEM of Formulation $C$ during long-term in vitro release $\left(37^{\circ} \mathrm{C}\right.$ ), at (a) day 0 (outer surface), (b) day 0 (higher magnification, fractured microspheres), and (c) day 180 (outer surface).

data at day 120 suggest that more than half the mass of the polymer matrix was lost due to polymer degradation and drug release. By day 180, when drug release was complete, only $20-30 \%$ of the polymer mass remained.

Figure 4 shows the scanning electron micrographs (SEM) of Formulation $C$ at days 0 and 180. Microspheres of Formulation $C$ were spherical in the range of $13-18 \mu \mathrm{m}$, with a mean diameter of approximately $15.9 \mu \mathrm{m}$. The outer surface of the microspheres was relatively smooth with a few small nonuniform pores of varying sizes (Figure 4(a)). The intact microspheres fractured with some difficulty showing a porous interior (Figure 4(b)). By day 180, when release was complete and only $20-30 \%$ mass remained, the remnant polymer matrix fragments existed as smaller sized particles, several of them which were non-spherical and porous (Figure 4(c)). Thus, the SEM images corroborate the mass loss data (Figure 3).

3.5. Correlating Long-Term In Vitro Release with Mass Loss and Hydration. A comparison of the data from Figures 1 to 4 confirms the triphasic drug release mechanism from Orntide PLA microspheres. The low initial burst values suggest that most of the drug was encapsulated into the polymer matrix, rather than the outer surface or readily accessible pores. This fact is easily explained by long-term in vitro release profile of the formulations, where drug release was well controlled by the polymer for a period of over 6 months. The steady diffusional phase of release, a consequence of polymer hydration, can be explained by correlating the hydration data (Figure 2) with the scanning electron micrograph (Figures $4(\mathrm{a})$ and $4(\mathrm{~b}))$. Given that a porous network was observed on the outer and inner surface of the microspheres, there are few barriers for water incursion into the polymer as well as drug release from the polymer to the outer sink. This explains the rapid and consistent hydration data (Figure 2) and drug release (Figure 1) during the diffusional phase. Another consequence of the porous nature of the microspheres is the ease of access to the polymer chains, allowing water molecules to initiate hydrolytic degradation of the ester bonds in the polyester based polymer. Breakdown of the polymer chains leads to formation of acidic functionalities inside the polymer, some of which are responsible for autocatalysis that is a characteristic of the erosional phase of drug release. Indeed, the mass loss data (Figure 3 ) suggest that the porous microspheres were rapidly hydrated leading to drug release and polymer breakdown in a steady fashion.

3.6. Accelerated In Vitro Release Experiments. Figures 5-7 show the results of short-term (accelerated) release at $51^{\circ} \mathrm{C}$, $55^{\circ} \mathrm{C}$, and $59^{\circ} \mathrm{C}$, respectively, for Formulations $A-C$. At first glance, the plots in Figures 5-7 appear biphasic, different from the long-term release profile detailed in Figure 1. Further, the plots show a clear temperature dependency (Figure 8).

At $51^{\circ} \mathrm{C}$, the microspheres show an initial burst followed by steady diffusional phase of drug release (Figure 5). However, drug release rate appears to slow down and plateau after day 2 through day 9 , after which the short-term study was terminated. Mass balance of the microspheres at day 9 revealed that the unreleased drug was entrapped inside the microspheres (data not shown). Increasing the temperature to $55^{\circ} \mathrm{C}$ resulted in a biphasic profile similar to that observed at $51^{\circ} \mathrm{C}$, except that the plateau occurred after $50-60 \%$ drug release had occurred (Figure 6). Once again, mass balance results confirmed that the remaining drug was present inside the polymer matrix (data not shown). Further elevation of the temperature to $59^{\circ} \mathrm{C}$ (Figure 7) showed a biphasic profile similar to $51^{\circ} \mathrm{C}$ and $55^{\circ} \mathrm{C}$, except that complete release was achieved by day 4 . 


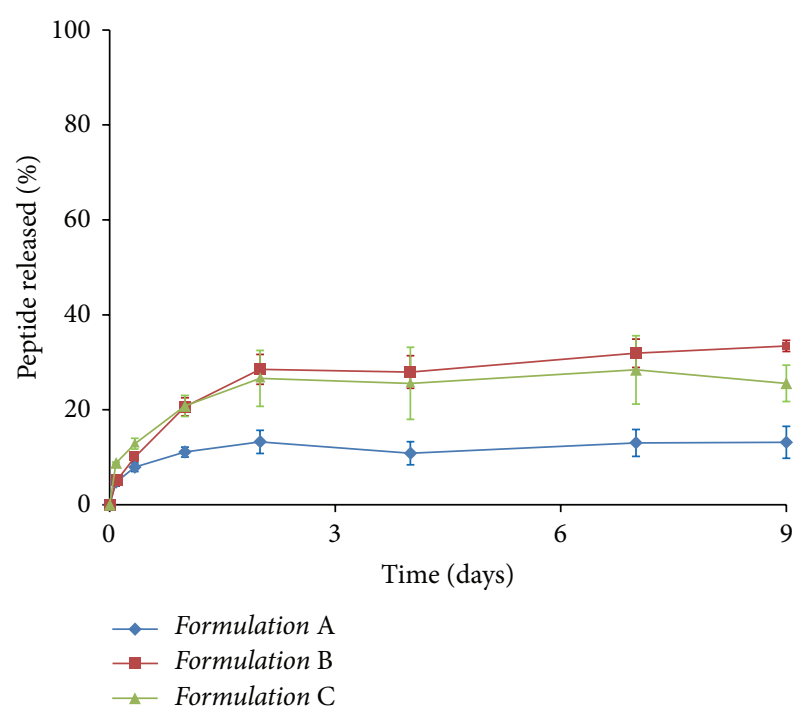

FIGURE 5: Short-term in vitro release of Orntide PLA microspheres $\left(51^{\circ} \mathrm{C}\right)$.

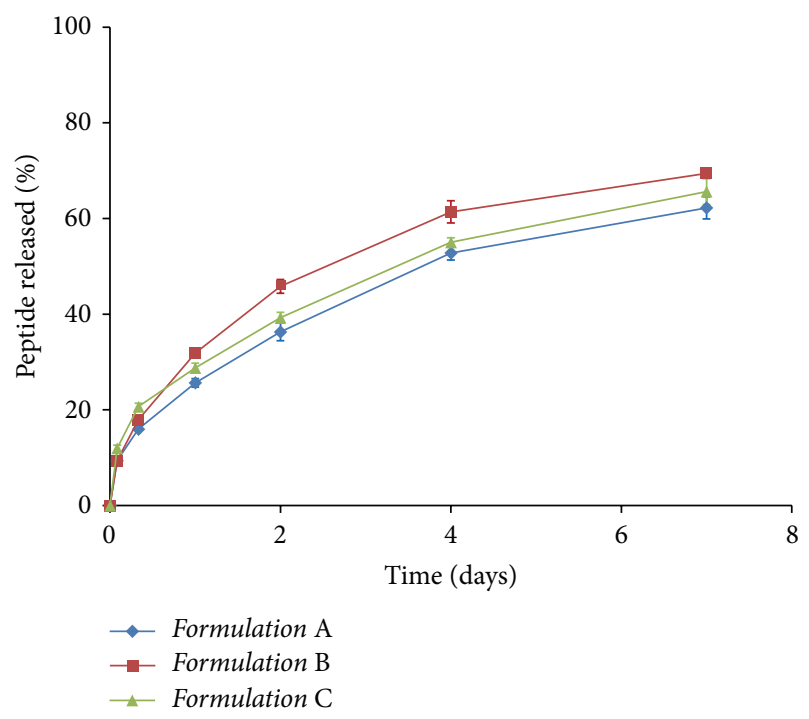

FIGURE 6: Short-term in vitro release of Orntide PLA microspheres $\left(55^{\circ} \mathrm{C}\right)$.

The effect of temperature on the drug release rate and profile for Formulation $C$ is shown in Figure 8. As noted earlier, similar biphasic profiles were observed at the three elevated temperatures investigated, with a plateau observed at the two lower temperatures.

The biphasic short-term drug release profiles from Figures 5-8 indicated a change in release mechanism at the elevated temperatures. Indeed, the profiles suggested that drug release occurred predominantly by diffusion, not erosion, and is evidenced by the similar nature in release behavior at all the temperatures studied. This change in release mechanism was unexpected and varied from previous reports from our group where short-term release profiles correlated well with long-term drug release behavior, with no change in

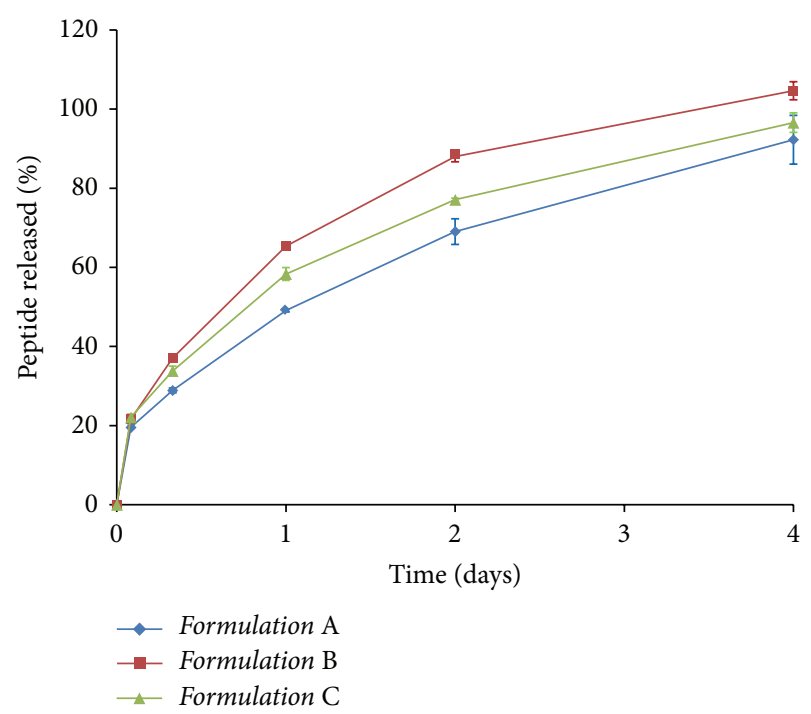

FIGURE 7: Short-term in vitro release of Orntide PLA microspheres $\left(59^{\circ} \mathrm{C}\right)$.

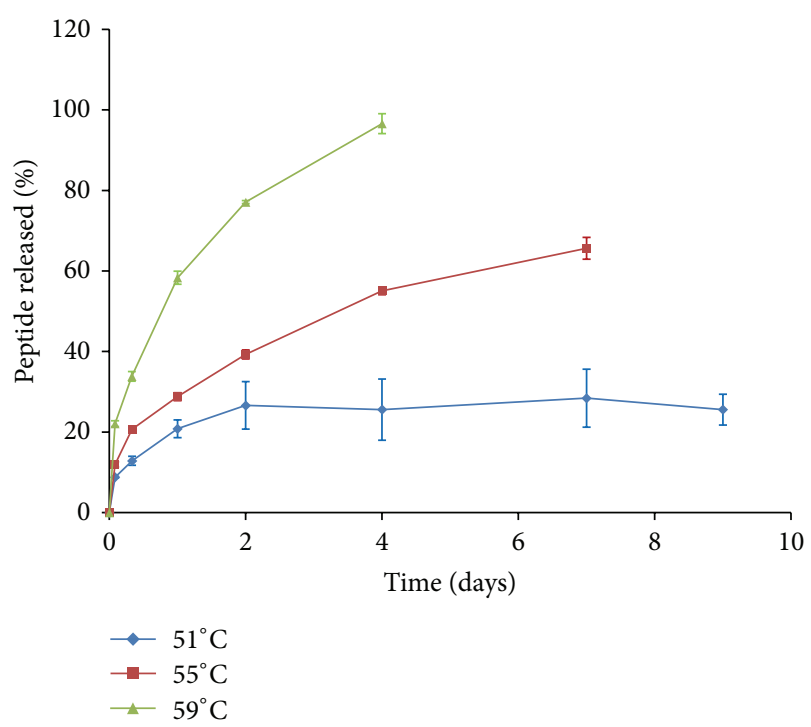

FIGURE 8: Effect of temperature on short-term in vitro release of Formulation $C$.

release mechanisms [44-46]. Additional experiments will be required to investigate and understand the findings of the short-term release behavior of Orntide PLA microspheres.

Nevertheless, Figures 5-7 clearly show that short-term release at the elevated temperatures occurred by diffusion of drug from the polymer matrix to the outer sink. Analysis of the release profiles by applying the Higuchi equation revealed an excellent goodness of fit $\left(R^{2}\right.$ between 0.95 and 0.99 ) for all the formulations at all the temperatures evaluated, confirming that short-term drug release occurred by diffusion (Figures 9, 10, and 11). Indeed, a linear relationship between drug release and the square root of time is a distinct 


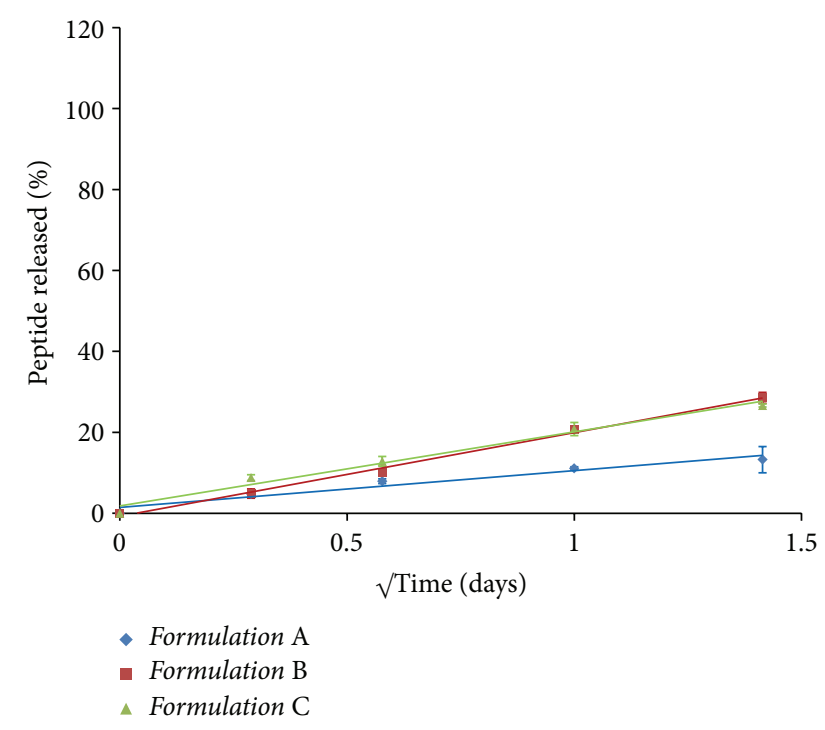

FIGURE 9: Higuchi plot for short-term in vitro release of Orntide PLA microspheres $\left(51^{\circ} \mathrm{C}\right)$.

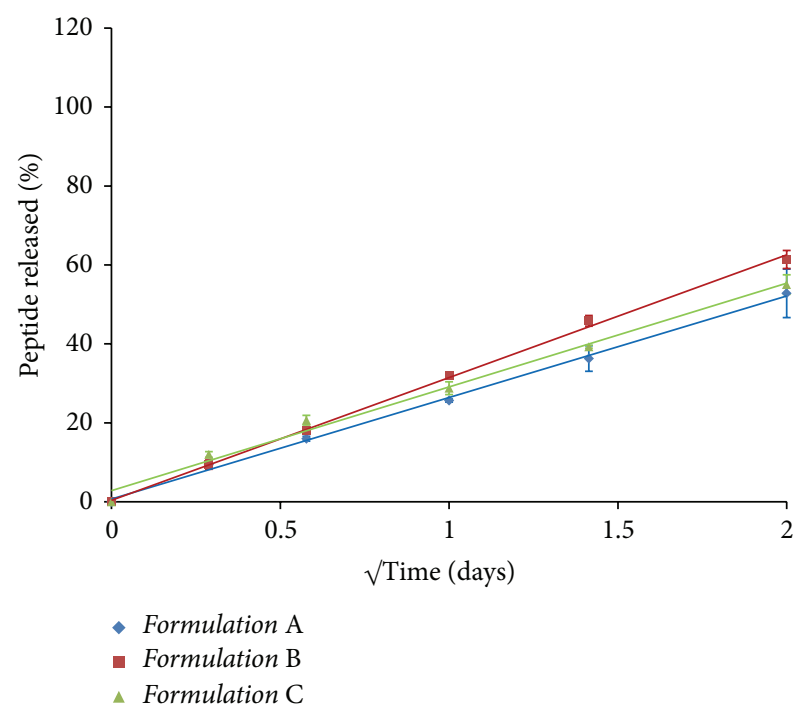

FIGURE 10: Higuchi plot for short-term in vitro release of Orntide PLA microspheres $\left(55^{\circ} \mathrm{C}\right)$.

characteristic of drug delivery systems that follow diffusional release [47] and can be described by the following equation:

$$
\mathrm{Q}=K_{H} \times t^{1 / 2},
$$

where " $Q$ " = cumulative drug release at time " $t$ " and " $K_{H}$ " = rate constant $=$ slope of the line obtained by plotting cumulative drug release against the square of time (Figures 9-11). Values of $K_{H}$ were dependent on temperature and ranged from 10 to 20,25 to 30 , and 45 to $55 \%$ release $/$ day ${ }^{1 / 2}$ at $51^{\circ} \mathrm{C}$, $55^{\circ} \mathrm{C}$, and $59^{\circ} \mathrm{C}$, respectively.

Since drug release at the elevated temperatures showed a clear temperature dependency, the energetics of diffusional release was calculated using the Arrhenius equation, as

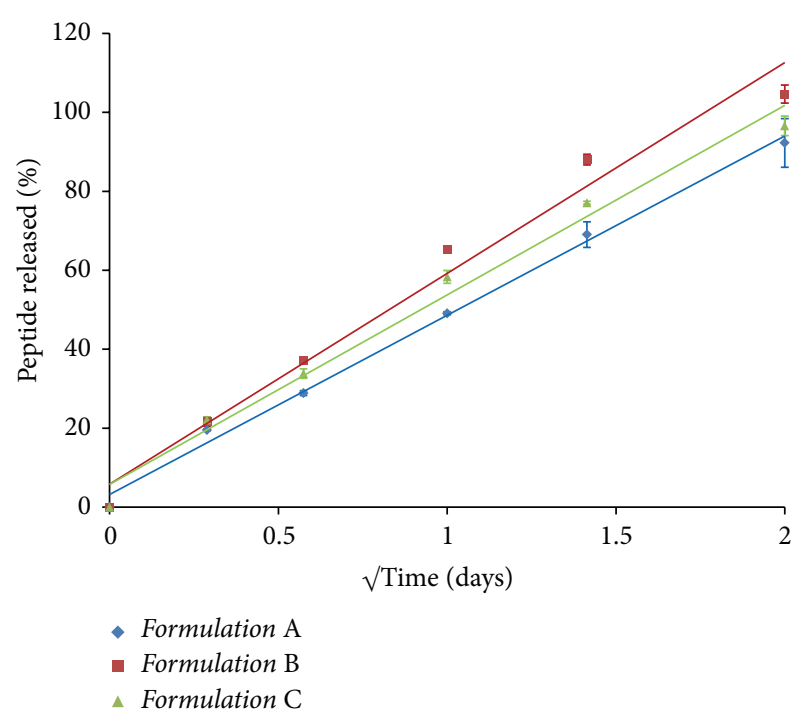

FIGURE 11: Higuchi plot for short-term in vitro release of Orntide PLA microspheres $\left(59^{\circ} \mathrm{C}\right)$.

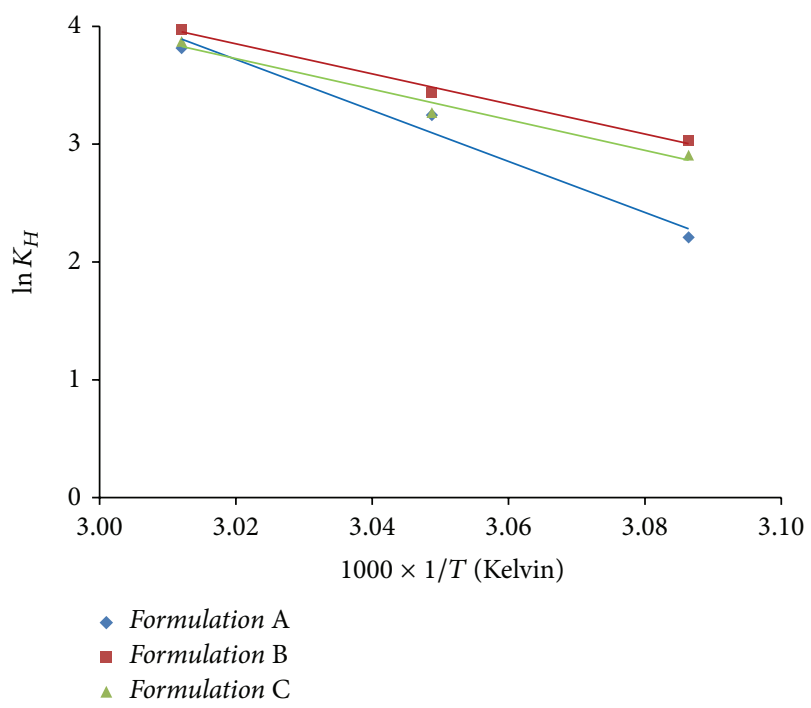

Figure 12: Arrhenius plot for Orntide PLA microspheres.

previously reported [45]. The Arrhenius equation describes the relationship between activation energy $\left(E_{a}\right)$, absolute temperature $(T)$, and the specific rate constant $(k)$ at that temperature.

$$
k=A e^{-E a / R T},
$$

where " $A$ " is a constant and " $R$ " is the universal gas constant $(1.987 \mathrm{cal} / \mathrm{mol})$.

Figure 12 and Table 2 show the results of Arrhenius treatment for the three Orntide PLA formulations. From Figure 12, a linear relationship was obtained when the natural logarithm of $K_{H}$ was plotted against the inverse absolute temperature $(T)$. Using the Arrhenius equation, the energy of activation for the diffusional phase of release was calculated to be approx $43 \mathrm{kcal} / \mathrm{mol}$ for Formulation $A$ and around 
TABLE 2: Results of Arrhenius treatment on Orntide PLA microspheres.

\begin{tabular}{lccc}
\hline & \multicolumn{3}{c}{ Formulation } \\
& $\mathrm{A}$ & $\mathrm{B}$ & $\mathrm{C}$ \\
\hline $\ln A$ & 69.10 & 42.38 & 42.91 \\
$E_{a}(\mathrm{kcal} / \mathrm{mol})$ & 43.02 & 25.35 & 25.78 \\
$R^{2}$ & 0.97 & 0.99 & 0.98 \\
\hline
\end{tabular}

$25 \mathrm{kcal} / \mathrm{mol}$ for Formulations $B$ and $C$ (Table 2) $\left(R^{2}\right.$ between 0.97 and 0.99). The values obtained for Formulations $B$ and $C$ are slightly lower than those reported previously for drug diffusion of Leuprolide from PLGA microspheres, where the lower $E_{a}$ values for PLGA polymer hydration confirmed that it was the rate limiting step in drug release from a PLGA matrix [46]. The lower $E_{a}$ values for Formulations $B$ and $C$ also indicate that $E_{a}$ for drug release from the slow degrading PLA polymer is greater than the faster degrading PLGA polymer. Additionally, the lower $E_{a}$ values suggest that diffusional processes in microspheres having a high drug to polymer ratio (Formulations $B$ and $C$ ) are faster than those where the drug loading is lower (Formulation $A$ ). Thus, the high $E_{a}$ value for Formulation $A$ containing a lower concentration of peptide molecules in the polymer matrix was not surprising as the energetics for the peptide molecules to diffuse through the PLA polymer would be greater.

Overall, the results of the current study suggest that by proper selection of a polyester polymer like PLA, drug release can be tailored to extend up to a period of 6 months or longer. Indeed, the PLA polymer with its low hydration and erosion rates is a suitable excipient to encapsulate Orntide, a watersoluble GnRH antagonist, and deliver sustained drug levels. From the perspective of a clinician and patient, such type of a dosage form will serve to reduce the frequency of dosing and enhance adherence to prostate cancer therapy leading to better healthcare outcomes.

\section{Conclusions}

Administration of a long-acting injectable dosage form of a peptide GnRH antagonist is an excellent approach to obtain sustained drug levels over an extended interval. In the current study, three formulations of Orntide, a GnRH antagonist, were prepared by encapsulation of the peptide into the slow degrading PLA polymer with the objective of providing clinicians an alternate treatment option for prostate cancer patients who may require extended therapy. Longterm in vitro drug release studies revealed that the Orntide PLA formulations would be suitable for 6-month dosing, indicating the suitability of using the slow degrading PLA homopolymer to allow for drug release over a prolonged interval. Thus, proper selection of biodegradable polyester polymer will provide the pharmaceutical scientist the ability to deliver poorly bioavailable peptide drugs as well as design drug delivery systems that are clinically relevant. Further, such an approach will allow for a decrease in the frequency of dosing and improve adherence to therapy in prostate cancer patients.

\section{Conflict of Interests}

The authors declare that there is no conflict of interests.

\section{Acknowledgments}

The research described in this paper was performed while the authors were affiliated with the University of Kentucky, Lexington, KY. The authors wish to thank Oakwood Labs, Oakwood, OH, and the Graduate School, University of Kentucky, Lexington, KY, for their financial support.

\section{References}

[1] J. Xuan, Y. Lin, J. Huang et al., "Exenatide-loaded PLGA microspheres with improved glycemic control: in vitro bioactivity and in vivo pharmacokinetic profiles after subcutaneous administration to SD rats," Peptides, vol. 46, pp. 172-179, 2013.

[2] J. He, M. Feng, X. Zhou et al., "Stabilization and encapsulation of recombinant human erythropoietin into PLGA microspheres using human serum albumin as a stabilizer," International Journal of Pharmaceutics, vol. 416, no. 1, pp. 69-76, 2011.

[3] L. Sun, S. Zhou, W. Wang, X. Li, J. Wang, and J. Weng, "Preparation and characterization of porous biodegradable microspheres used for controlled protein delivery," Colloids and Surfaces A: Physicochemical and Engineering Aspects, vol. 345, no. 1-3, pp. 173-181, 2009.

[4] H. K. Kim and T. G. Park, "Comparative study on sustained release of human growth hormone from semi-crystalline poly(L-lactic acid) and amorphous poly(D,L-lactic-co-glycolic acid) microspheres: morphological effect on protein release," Journal of Controlled Release, vol. 98, no. 1, pp. 115-125, 2004.

[5] T. G. Park, H. Y. Lee, and Y. S. Nam, "A new preparation method for protein loaded poly(D,L-lactic-co-glycolic acid) microspheres and protein release mechanism study," Journal of Controlled Release, vol. 55, no. 2-3, pp. 181-191, 1998.

[6] A. K. Banga and Y. W. Chien, "Systemic delivery of therapeutic peptides and proteins," International Journal of Pharmaceutics, vol. 48, no. 1-3, pp. 15-50, 1988.

[7] H. Okada and H. Toguchi, "Biodegradable microspheres in drug delivery," Critical Reviews in Therapeutic Drug Carrier Systems, vol. 12, no. 1, pp. 1-99, 1995.

[8] S. D’Souza, J. Faraj, and P. DeLuca, "Microsphere delivery of Risperidone as an alternative to combination therapy," European Journal of Pharmaceutics and Biopharmaceutics, vol. 85, pp. 631639, 2013.

[9] C. Garner, "Uses of GnRH agonists," Journal of Obstetric, Gynecologic, and Neonatal Nursing, vol. 23, no. 7, pp. 563-570, 1994.

[10] J. N. Pace, J. L. Miller, and L. I. Rose, "GnRH agonists: gonadorelin, leuprolide and nafarelin," The American Family Physician, vol. 44, no. 5, pp. 1777-1782, 1991.

[11] H. Lepor and N. D. Shore, "LHRH agonists for the treatment of prostate cancer: 2012," Reviews in Urology, vol. 14, pp. 1-12, 2012.

[12] P. M. Conn and W. F. Crowley Jr., "Gonadotropin-releasing hormone and its analogs," Annual Review of Medicine, vol. 45, pp. 391-405, 1994.

[13] E. Kienle and G. Lübben, "Efficacy and safety of leuprorelin acetate depot for prostate cancer," Urologia Internationalis, vol. 56, no. 1, pp. 23-30, 1996. 
[14] A. B. Copperman and C. Benadiva, "Optimal usage of the GnRH antagonists: a review of the literature," Reproductive Biology and Endocrinology, vol. 11, no. 1, article 20, 2013.

[15] J. A. F. Huirne and C. B. Lambalk, "Gonadotropin-releasinghormone-receptor antagonists," The Lancet, vol. 358, no. 9295, pp. 1793-1803, 2001.

[16] G. F. Weinbauer and E. Nieschlag, "LH-RH antagonists: state of the art and future perspectives," Recent Results in Cancer Research, vol. 124, pp. 113-136, 1992.

[17] L. A. Kiesel, A. Rody, R. R. Greb, and A. Szilágyi, “Clinical use of GnRH analogues," Clinical Endocrinology, vol. 56, no. 6, pp. 677-687, 2002.

[18] J. W. Kostanski, B. A. Dani, B. Schrier, and P. P. DeLuca, "Effect of the concurrent LHRH antagonist administration with a LHRH superagonist in rats," Pharmaceutical Research, vol. 17, no. 4, pp. 445-450, 2000.

[19] H. Okada, "One- and three-month release injectable microspheres of the LH-RH superagonist leuprorelin acetate," Advanced Drug Delivery Reviews, vol. 28, no. 1, pp. 43-70, 1997.

[20] R. Sharifi, L. D. Knoll, J. Smith, and E. Kramolowsky, "Leuprolide acetate (30-mg depot every four months) in the treatment of advanced prostate cancer," Urology, vol. 51, no. 2, pp. 271-276, 1998.

[21] S. L. Gupta, K. P. Rao, K. P. R. Choudary, and S. Pratima, "Preformulation studies of biodegradable drug implants of meloxicam for orthopedic patient care," Journal of Pharmaceutical Science and Technology, vol. 3, pp. 494-498, 2011.

[22] S. Takada, T. Kurokawa, K. Miyazaki, S. Iwasa, and Y. Ogawa, "Utilization of an amorphous form of a water-soluble GPIIb/ IIIa antagonist for controlled release from biodegradable microspheres," Pharmaceutical Research, vol. 14, no. 9, pp. 1146-1150, 1997.

[23] J. M. Ramstack, G. P. Grandolfi, E. Mannaert, P. D'Hoore, and R. A. Lasser, "Risperdal Consta: Prolonged-Release Injectable Delivery of Risperidone using Medisorb Microsphere Technology," Abstract AAPS, 2002.

[24] E. Comets, F. Mentré, F. Nimmerfall et al., "Nonparametric analysis of the absorption profile of octreotide in rabbits from long-acting release formulation OncoLAR," Journal of Controlled Release, vol. 59, no. 2, pp. 197-205, 1999.

[25] G.-K. Zou, Y.-L. Song, W. Zhou et al., "Effects of local delivery of bFGF from PLGA microspheres on osseointegration around implants in diabetic rats," Oral Surgery, Oral Medicine, Oral Pathology and Oral Radiology, vol. 114, no. 3, pp. 284-289, 2012.

[26] L. C. Amann, M. J. Gandal, R. Lin, Y. Liang, and S. J. Siegel, “in vitro-in vivo correlations of scalable plga-risperidone implants for the treatment of schizophrenia," Pharmaceutical Research, vol. 27, no. 8, pp. 1730-1737, 2010.

[27] U. Seju, A. Kumar, and K. K. Sawant, "Development and evaluation of olanzapine-loaded PLGA nanoparticles for nose-tobrain delivery: in vitro and in vivo studies," Acta Biomaterialia, vol. 7, no. 12, pp. 4169-4176, 2011.

[28] S. D’Souza, J. A. Faraj, S. Giovagnoli, and P. P. DeLuca, "Development of risperidone PLGA microspheres," Journal of Drug Delivery, vol. 2014, Article ID 620464, 11 pages, 2014.

[29] J. Braunecker, M. Baba, G. E. Milroy, and R. E. Cameron, “The effects of molecular weight and porosity on the degradation and drug release from polyglycolide," International Journal of Pharmaceutics, vol. 282, no. 1-2, pp. 19-34, 2004.
[30] T. G. Park, "Degradation of poly(lactic-co-glycolic acid) microspheres: effect of copolymer composition," Biomaterials, vol. 16, no. 15, pp. 1123-1130, 1995.

[31] E. O. Akala, P. Wiriyacoonkasem, and G. Pan, "Studies on in vitro availability, degradation, and thermal properties of naltrexone-loaded biodegradable microspheres," Drug Development and Industrial Pharmacy, vol. 37, no. 6, pp. 673-684, 2011.

[32] S. D’Souza, J. A. Faraj, S. Giovagnoli, and P. P. DeLuca, "Preparation, characterization and in vivo evaluation of Olanzapine Poly(D, L-lactide-co-glycolide) (PLGA) microspheres," Journal of Pharmaceutics, vol. 2013, Article ID 831381, 9 pages, 2013.

[33] J. W. Kostanski, B. C. Thanoo, and P. P. DeLuca, "Preparation, characterization, and in vitro evaluation of 1- and 4-month controlled release orntide PLA and PLGA microspheres," Pharmaceutical Development and Technology, vol. 5, no. 4, pp. 585596, 2000.

[34] G. Ploussard and P. Mongiat-Artus, "Triptorelin in the management of prostate cancer," Future Oncology, vol. 9, no. 1, pp. 93102, 2013

[35] C.-C. Kao, Y.-H. Chang, T. Wu et al., "Open, multi-center, phase IV study to assess the efficacy and tolerability of triptorelin in Taiwanese patients with advanced prostate cancer," Journal of the Chinese Medical Association, vol. 75, no. 6, pp. 255-261, 2012.

[36] D. Fontana, M. Mari, A. Martinelli et al., "3-Month formulation of goserelin acetate ("Zoladex" 10.8-mg Depot) in advanced prostate cancer: results from an Italian, open, multicenter trial," Urologia Internationalis, vol. 70, no. 4, pp. 316-320, 2003.

[37] E. David Crawford and J. M. Phillips, "Six-month gonadotropin releasing hormone $(\mathrm{GnRH})$ agonist depots provide efficacy, safety, convenience, and comfort," Cancer Management and Research, vol. 3, no. 1, pp. 201-209, 2011.

[38] U. W. Tunn, "A 6-month depot formulation of leuprolide acetate is safe and effective in daily clinical practice: a noninterventional prospective study in 1273 patients," BMC Urology, vol. 11, article 15, 2011.

[39] A. Spitz, J. M. Young, L. Larsen, C. Mattia-Goldberg, J. Donnelly, and K. Chwalisz, "Efficacy and safety of leuprolide acetate 6-month depot for suppression of testosterone in patients with prostate cancer," Prostate Cancer and Prostatic Diseases, vol. 15, no. 1, pp. 93-99, 2012.

[40] U. W. Tunn, D. Gruca, and P. Bacher, "Six-month leuprorelin acetate depot formulations in advanced prostate cancer: a clinical evaluation," Clinical Interventions in Aging, vol. 8, pp. 457-464, 2013.

[41] J. W. Kostanski and P. P. DeLuca, "A novel in vitro release technique for peptide containing biodegradable microspheres," AAPS PharmSciTech, vol. 1, no. 1, article E4, 2000.

[42] S. D’Souza, J. A. Faraj, S. Giovagnoli, and P. P. DeLuca, "IVIVC from long acting olanzapine microspheres," International Journal of Biomaterials, vol. 2014, Article ID 407065, 11 pages, 2014.

[43] G. Reich, "Use of DSC to study the degradation behavior of PLA and PLGA microparticles," Drug Development and Industrial Pharmacy, vol. 23, no. 12, pp. 1177-1189, 1997.

[44] M. Shameem, H. Lee, and P. P. DeLuca, "A short-term (accelerated release) approach to evaluate peptide release from PLGA depot formulations," AAPS PharmSci, vol. 1, no. 3, article 7, 1999.

[45] S. D’Souza, J. A. Faraj, R. Dorati, and P. P. DeLuca, "A short term quality control tool for biodegradable microspheres," AAPS PharmSciTech, vol. 15, no. 3, pp. 530-541, 2014. 
[46] S. S. D'Souza, J. A. Faraj, and P. P. DeLuca, "A model-dependent approach to correlate accelerated with real-time release from biodegradable microspheres," AAPS PharmSciTech, vol. 6, no. 4, article 70, 2005.

[47] T. Higuchi, "Rate of release of medicaments from ointment bases containing drugs in suspension," Journal of pharmaceutical sciences, vol. 50, pp. 874-875, 1961. 

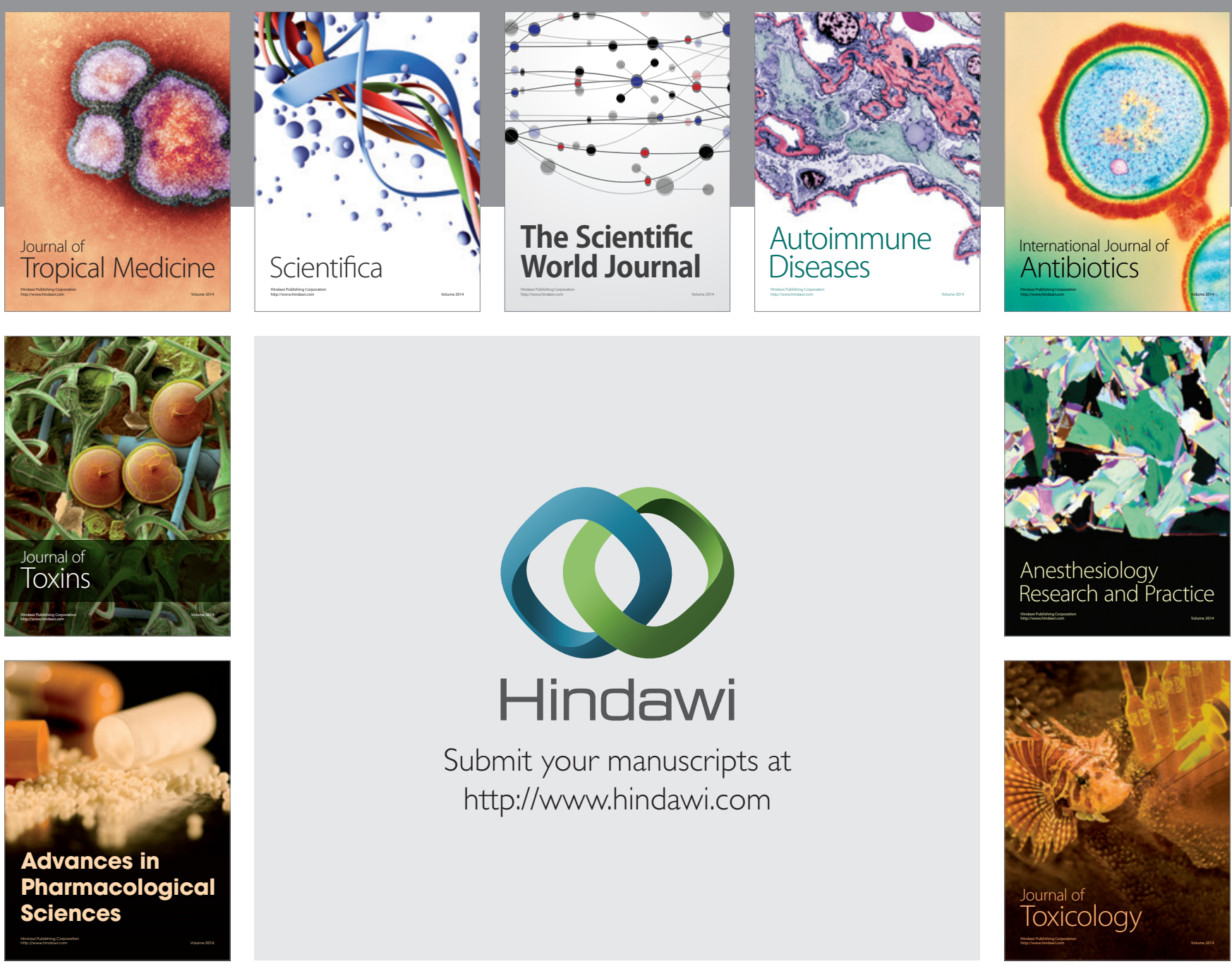

\section{Hindawi}

Submit your manuscripts at

http://www.hindawi.com
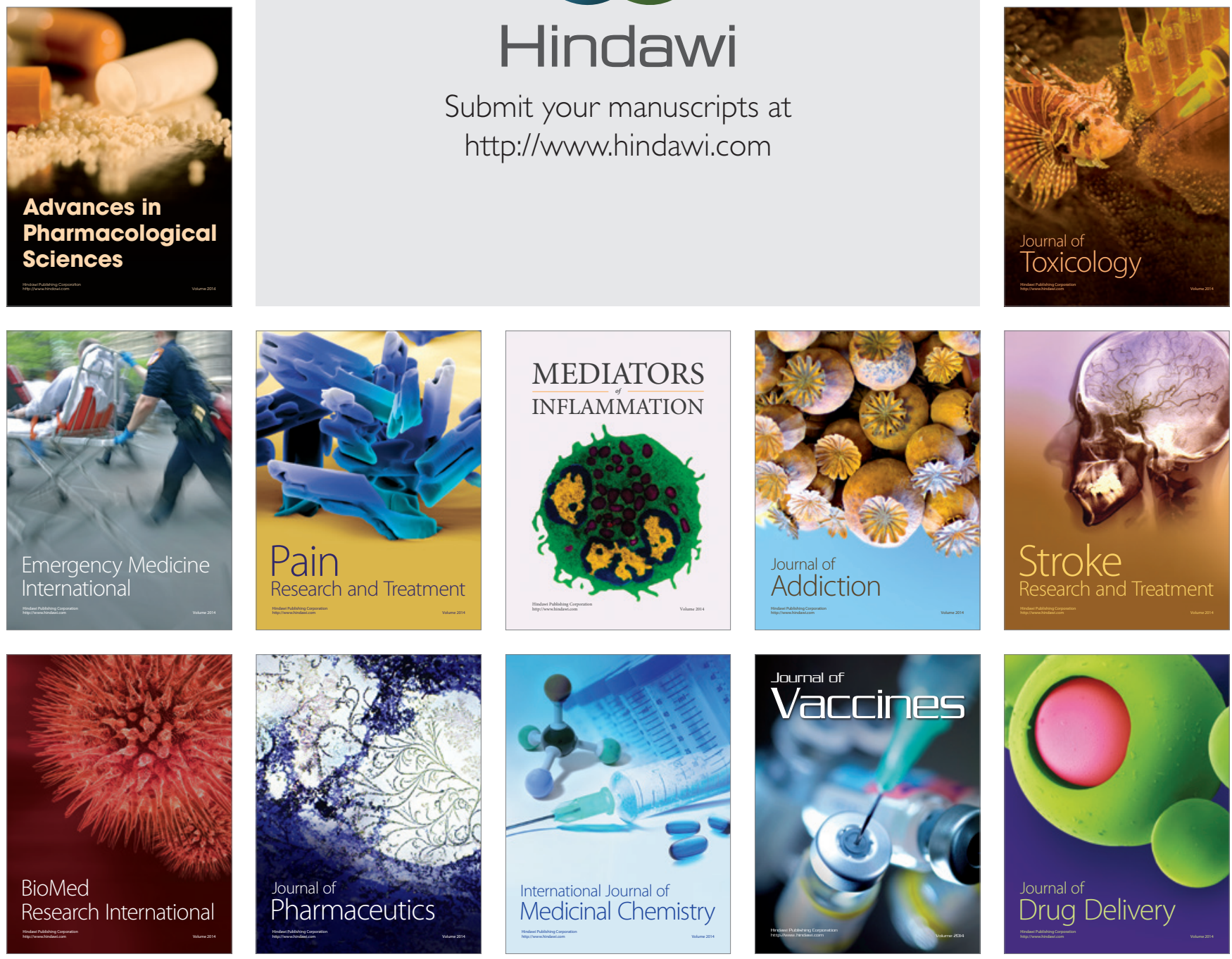